\title{
Linear Covariance Analysis for a Lunar Lander
}

\author{
Jiann-Woei Jang ${ }^{1}$, Sagar Bhatt ${ }^{2}$, Matthew Fritz ${ }^{3}$ and David Woffinden ${ }^{4}$ \\ The Charles Stark Draper Laboratory, Inc., Houston, TX, 77058 \\ Darryl May ${ }^{5}$, Ellen Braden ${ }^{6}$ \\ NASA Johnson Space Center, Houston, TX 77058 \\ Michael Hannan ${ }^{7}$ \\ NASA Marshall Space Flight Center, Huntsville, AL 35812
}

\begin{abstract}
A next-generation lunar lander Guidance, Navigation, and Control (GNC) system, which includes a state-of-the-art optical sensor suite, is proposed in a concept design cycle. The design goal is to allow the lander to softly land within the prescribed landing precision. The achievement of this precision landing requirement depends on proper selection of the sensor suite. In this paper, a robust sensor selection procedure is demonstrated using a Linear Covariance (LinCov) analysis tool developed by Draper.
\end{abstract}

\section{Introduction}

Since the Lunar Reconnaissance Orbiter found evidence of water on the Moon's surface in 2009, the excitement over the implications of water on the Moon for future human missions has triggered multiple moon exploration proposals. For example, the Resource Prospector (RP) mission is an in-situ resource utilization (ISRU) technology demonstration mission under study by NASA ${ }^{1}$. Future moon missions like RP will conduct activities to characterize the conditions of representative exploration environments, identify hazards, and assess resources to enable future human exploration ${ }^{1}$.

Some near-term lunar exploration mission proposals have one common goal, which is to verify the existence of hydrogen. To maximize the probability of mission success, precision lunar landing is the ultimate mission requirement. The entire lunar mission can be broken into the following sub-phases of flight: Earth ascent, Low Earth Orbit circularization, translunar insertion, translunar cruise, lunar orbit insertion, lunar orbit circularization, lunar descent insertion, braking, coasting, and final descent and landing. Each segment between those waypoints requires precision flight control to ensure states at the end of each segment are achievable. Control precision is dependent on prudent sensor suite design. Linear Covariance (LinCov) analysis ${ }^{2}$, using a statistical approach to "envelope" the Monte-Carlo results in a fraction of the time, has been used to design sensor suite for powered lunar descent and landing ${ }^{3}$. LinCov analysis enables a program to trade many combinations of sensors early in the design phase and search for sensor combinations that reduce cost while meeting requirements.

This paper studies the final lunar descent phases of the lunar exploration mission, starting from the braking phase through the terminal descent phase and ending with landing on the surface of the moon. A conceptual GNC design is used to illustrate a candidate final lunar descent profile. The conceptual GNC scheme will be briefly introduced. Then an overview of the candidate sensor models will be presented. A brief overview of linear covariance theory and LinCov analysis tool utilized in this research will be provided. A linear covariance analysis will then be performed for the final descent phase. Finally, a robust sensor selection procedure is discussed.

${ }^{1}$ Principal Member of the Technical Staff; Draper, Houston TX, 77058: jang@ draper.com, AIAA Senior Member ${ }^{2}$ Senior Member of the Technical Staff; Draper, Cambridge MA, 02139: sbhatt@ draper.com

${ }^{3}$ Member of the Technical Staff; Draper, Houston TX, 77058: mfritz@ draper.com

${ }^{4}$ Senior Member of the Technical Staff; Draper, Houston TX, 77058: dwoffinden@ draper.com

${ }^{5}$ Aerospace Engineer, NASA Johnson Space Center, Houston, TX 77058: darryl.w.may@ nasa.gov

${ }^{6}$ Aerospace Engineer, NASA Johnson Space Center, Houston, TX 77058: ellen.m.braden@nasa.gov

${ }^{7}$ Aerospace Engineer, NASA Marshall Space Flight Center, Huntsville, AL, 35812: mike.r.hannan@ nasa.gov 


\section{Lunar Descent GNC}

In this section, the lunar descent GNC architecture is briefly outlined. The conceptual GNC mission assumes that the lunar descent GNC mission consists of three segments: braking, coast, and terminal descent and landing. The braking phase of flight starts from the initial descent altitude and velocity and uses a solid booster to significantly reduce the lander speed before the lander transitions into the coast phase. GNC consists of navigation, guidance, and control subsystems. The navigation module processes sensed data from various sensors and outputs estimated translational and rotational states for guidance and control to use. An example sensor suite is detailed in the next section. The guidance module uses the translational states from the navigation module along with the target landing site state to calculate the desired translational acceleration. In this work, the lunar powered descent guidance is divided into three segments: (1) Braking Phase, (2) Coast Phase, and (3) Approach and Landing Phase. An Apollo explicit guidance law is used as the descent guidance $\operatorname{logic}^{4}$ as an example. The commanded acceleration $\mathbf{a}_{\mathbf{c}}$ is defined as in Eq. (1)

$$
\mathbf{a}_{\mathbf{c}}=\frac{12}{T^{2}}\left(\mathbf{r}_{\mathbf{t}}-\mathbf{r}\right)+\frac{6}{T}\left(\mathbf{v}_{\mathbf{t}}+\mathbf{v}\right)+\mathbf{a}_{\mathbf{t}}
$$

where $T$ is the guidance time, $\mathbf{r}$ and $\mathbf{v}$ are the current position and velocity states, and $\mathbf{r}_{\mathbf{t}}, \mathbf{v}_{\mathbf{t}}$, and $\mathbf{a}_{\mathbf{t}}$ are the targeted position, velocity and acceleration, respectively. The steering algorithm in the guidance module calculates the desired attitude states for control. In the control module, attitude and rate errors are calculated by subtracting the navigation-estimated attitude/rate from the commanded attitude/rate. The resulting rotational states then are filtered by flex filters before they are sent to attitude control subsystem. During the braking phase, the example lander uses a Thrust Vector Control (TVC) system for pitch and yaw control and Reaction Control System (RCS) for roll control. The TVC controller consists of both Proportional-Integral-Derivative (PID) control and load relief control. The PID controller is designed using gain scheduling, a process that involves optimizing a controller for specific design points throughout the time of flight and linearly interpolating between these points to obtain gain values for any flight time ${ }^{5}$. The load relief controller for a lander vehicle can be used to balance disturbances due to hardware imperfections or build tolerances resulting from biased thrust vectors, thrust vector uncertainty, and misaligned sensors. During the coast phase of flight, no thrust activity will be scheduled. Following the coast phase, the terminal descent phase will use throttleable liquid thrusters for the precision descent and landing; the pitch and yaw attitude errors are controlled by differentially firing opposing thrusters while roll attitude error is mitigated by RCS thrusters commanded via phase plane logic. The phase plane controller is an inherently nonlinear system which necessitates the use of nonlinear techniques in order to predict the system's behavior. The detailed design and analysis of the fixed-jets roll control system can be found in References 6 to 9 .

\section{Candidate Sensor Suites and Baseline Sensor Specifications}

The candidate sensor suite for the conceptual lunar mission is first baselined. The initial error assumptions for each sensor are specified first. The success of a precision landing will be reliant on the proper sensor selection which includes identifying the performance of the sensor suite as an integrated system. Throughout the final descent flight phase, an inertial measurement unit (IMU), consisting of both a rate gyro and an accelerometer, will be sampled at a high rate to provide both rotational and translation state estimation. During the coast phase of flight, a star tracker will be used for rotational state estimation. Two separate sensors will be activated based on a minimum altitude threshold, a Terrain Relative Navigation (TRN) sensor ${ }^{10,11}$ and a radar altimeter. The TRN sensor will be activated near the end of the coast phase whereas the radar altimeter will be activated during the terminal descent phase. The TRN sensor will be used to provide position state measurements whereas the radar altimeter will provide attitude state updates. The TRN sensor will be shut down at an altitude where the stored imagery resolution is no longer adequate. The altimeter will be shut down when thruster plume from the lunar lander kicks up substantial lunar regolith 20 to 30 meters above the surface. Dead reckoning using IMU only will be used during the last portion of the descent once the other sensors have been deactivated. A detailed explanation of each sensor as modeled and used for the LinCov analysis is discussed below. 


\section{$\underline{\text { IMU Sensor Model }}$}

As previously mentioned, the IMU ${ }^{12}$ consists of a translational measurement device (accelerometer) and a rotational measurement device (gyro). The IMU model perturbs the true translational acceleration and rotational rate by a combination of noise components to produce the sensor measurement. The accelerometer model is shown in Eq. (2) and the gyro model is shown in Eq. (3)

$$
\begin{gathered}
\tilde{\mathbf{a}}_{\text {case }}=\left(\mathbf{I}+\mathbf{S} \mathbf{F}_{\mathbf{a}}+\mathbf{E}_{\mathrm{a}}+\boldsymbol{\Theta}_{\mathrm{a}}\right)\left(\mathbf{T}_{\mathbf{i}}^{\text {case }} \mathbf{a}_{\mathbf{i}}\right)+\mathbf{b}_{\mathbf{a}}+\mathbf{v}_{\mathrm{a}} \\
\tilde{\boldsymbol{\omega}}_{\text {case }}=\left(\mathbf{I}+\mathbf{S} \mathbf{F}_{\boldsymbol{\omega}}+\boldsymbol{\Theta}_{\boldsymbol{\omega}}\right)\left(\mathbf{T}_{\mathbf{i}}^{\text {case }} \boldsymbol{\omega}_{\mathbf{i}}\right)+\mathbf{b}_{\boldsymbol{\omega}}+\mathbf{v}_{\boldsymbol{\omega}}
\end{gathered}
$$

where I represents a 3x3 identity matrix, $\mathbf{S F}_{\mathbf{x}}$ represents a scale factor matrix, $\mathbf{E}_{\mathbf{a}}$ represents the non-orthogonality component, $\Theta_{\mathbf{x}}$ represents the internal misalignments, $\mathbf{T}_{\mathbf{i}}^{\text {case }}$ represents the direction cosine matrix (DCM) from an inertial fixed frame to the IMU case frame, $\mathbf{b}_{\mathbf{x}}$ represents the accelerometer/gyro bias and $\boldsymbol{v}_{\mathbf{x}}$ represents the noise present on the measurement.

\section{$\underline{\text { Star Tracker Sensor Model }}$}

The star tracker model ${ }^{13}$ perturbs the true attitude of the lander through a combination of alignment error and measurement noise. The star tracker produces a measurement of the lander attitude relative to the inertial reference frame as seen from the star tracker case frame. Since a star tracker is an image sensor which processes a star capture into an attitude estimate by comparing the image to an onboard star catalog, error from this internal estimation process will be present in the measurement. It is assumed this error is modeled via the measurement noise in the following model provided by Eq. (4)

$$
\widetilde{\mathbf{q}}_{\mathbf{i}}^{\text {st }}=\mathbf{q}_{\boldsymbol{\eta}} \otimes \mathbf{q}_{\mathbf{e}} \otimes \mathbf{q}_{\mathbf{b}}^{\text {st }} \otimes \mathbf{q}_{\mathbf{i}}^{\mathbf{b}}
$$

where $\widetilde{\mathbf{q}}_{\mathbf{i}}^{\text {st }}$ represents the inertial to star tracker case attitude, $\mathbf{q}_{b}^{\text {st }}$ represents the body to star tracker case attitude (which is a known quantity based on sensor mounting on the vehicle), $\mathbf{q}_{\mathbf{i}}^{\mathbf{b}}$ represents the inertial to body attitude, $\mathbf{q}_{\mathbf{e}}$ represents the misalignment error and $\mathbf{q}_{\boldsymbol{\eta}}$ represents the error due to the measurement noise.

\section{$\underline{\text { Radar Altimeter Sensor Model }}$}

The radar altimeter mode $\mathrm{l}^{11}$ corrupts the true altitude by measurement noise and an altimeter bias. While radar altimeters convert frequency shifts in radar signals into range estimates, the altimeter model implemented computes the altitude based on the position of the lander, which provides effectively the same information while simplifying the calculation. The altimeter model used during the LinCov analysis is shown in Eq. (5)

$$
\tilde{h}=h+b_{h}+v_{h}
$$

where $h$ represents the true altitude of the lander, $b_{h}$ represents the measurement bias and $v_{h}$ represents the noise present on the measurement.

\section{$\underline{\text { TRN Sensor Model }}$}

The TRN sensor ${ }^{10,11}$ is an image processing sensor that utilizes an onboard map of the landing site and overlays images captured during descent to determine the position of the lander relative to the surface. The actual implementation of an actual TRN sensor differs from the model implemented during the LinCov analysis. The TRN 
model implemented does not directly process imagery data into a position estimate since this is typically handled by a separate onboard algorithm. Instead, it is assumed that the measurement from the TRN sensor is the position estimated from this separate onboard algorithm. As a result of this assumption made to simplify the modeling of the TRN sensor, additional errors that are frequently captured by separate individual components within the TRN onboard position estimate algorithm are instead captured through an increase in the measurement noise which is typically provided by this separate algorithm. The TRN model used during the LinCov analysis is shown in Eq. (6)

$$
\tilde{\mathbf{r}}_{\text {trn/ref }}^{\mathbf{f}}=\mathbf{T}_{\mathrm{i}}^{\mathrm{f}} \mathbf{r}_{\text {trn }}^{\mathrm{i}}-\mathbf{T}_{\text {case }}^{\mathrm{f}} \mathbf{r}_{\text {ref }}^{\text {case }}+\mathbf{b}_{\text {trn }}+\mathbf{v}_{\text {trn }}
$$

where $\tilde{\mathbf{r}}_{\text {trn/ref }}^{\mathbf{f}}$ represents the position of the TRN sensor relative the reference position in the planet surface fixed frame, $\mathbf{r}_{\text {trn }}^{\mathrm{i}}$ represents the position of the TRN sensor in the inertial frame, $\mathbf{r}_{\text {ref }}^{\text {case }}$ represents the reference position in the TRN sensor case frame, $\mathbf{T}_{\mathbf{i}}^{\mathbf{f}}$ represents the DCM from inertial frame to planet surface fixed frame, $\mathbf{T}_{\text {case }}^{\mathbf{f}}$ represents the DCM from TRN sensor case frame to planet surface fixed frame, $\mathbf{b}_{\text {trn }}$ represents the measurement bias and $v_{\text {trn }}$ represents the noise present on the measurement.

Table 1 provides examples of candidate sensor suites for the conceptual lunar lander. As shown in Figure 1a, with radar altimeter measurements only, the navigation algorithm is able to estimate the vehicle's altitude state but not the downrange/cross-track states. In Figure 1b, with the TRN sensor included, the navigation algorithm can estimate all three directions, resulting in a drastic improvement in the overall RSS position error. The validity of this sensor suite will be verified via LinCov analysis and the corresponding results will be summarized in Section V.

\begin{tabular}{|c|c|c|c|c|c|c|c|c|}
\hline GYROS & $\begin{array}{c}\text { GYRO } \\
\text { NOISE } \\
\text { [deg/sqrt(hr)] 1- } \sigma\end{array}$ & 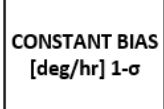 & $\begin{array}{l}\text { SCALE FACTOR } \\
\text { [ppm] 1- } \sigma\end{array}$ & 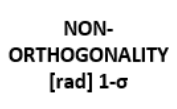 & $\begin{array}{l}\text { MARKOV } \\
\text { BIAS } \\
\text { TAU } \\
\text { [sec] }\end{array}$ & $\begin{array}{l}\text { MARKOV SS } \\
\text { BIAS } \\
\text { [deg/hr] 1- } \sigma\end{array}$ & $\begin{array}{l}\text { MARKOV SCALE } \\
\text { FACTOR TAU } \\
\text { [sec] }\end{array}$ & $\begin{array}{l}\text { MARKOV SS } \\
\text { SCALE FACTOR } \\
\text { [ppm] 1- } \sigma\end{array}$ \\
\hline LN200S & 0.07 & 0.10 & 100 & 20 & 100 & 0 & 100 & 0 \\
\hline
\end{tabular}

\begin{tabular}{|c|c|c|c|c|c|c|c|c|}
\hline ACCELEROMETER & $\begin{array}{l}\text { ACCELEROMETER } \\
\text { NOISE } \\
{[\mu \mathrm{g} / \mathrm{sqrt}(\mathrm{Hz})] \text { 1- } \sigma}\end{array}$ & $\begin{array}{c}\text { CONSTANT BIAS } \\
{[\mu \mathrm{g}] 1-\sigma}\end{array}$ & $\begin{array}{l}\text { SCALE FACTOR } \\
\text { [ppm] 1- } \sigma\end{array}$ & 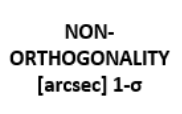 & $\begin{array}{c}\text { MARKOV } \\
\text { BIAS } \\
\text { TAU } \\
\text { [sec] }\end{array}$ & 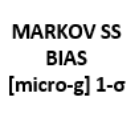 & $\begin{array}{l}\text { MARKOV SCALE } \\
\text { FACTOR TAU } \\
\text { [sec] }\end{array}$ & $\begin{array}{l}\text { MARKOV SS } \\
\text { SCALE FACTOR } \\
\text { [ppm] 1- } \sigma\end{array}$ \\
\hline LN200S & 35 & 300 & 300 & 20.0 & 60 & 0 & 100 & 0 \\
\hline
\end{tabular}

\begin{tabular}{|c|c|c|c|c|}
\hline STAR TRACKER & $\begin{array}{l}\text { BORESIGHT } \\
\text { [arcsec] 1- } \sigma\end{array}$ & $\begin{array}{l}\text { CROSS BORESIGHT } \\
\text { [arcsec] 1- } \sigma\end{array}$ & $\begin{array}{l}\text { MISALIGNMENT } \\
\text { [arcsec] 1- } \sigma\end{array}$ & $\begin{array}{l}\text { SAMPLE RATE } \\
\text { [sec] }\end{array}$ \\
\hline SODERN & 207 & 49 & 12 & 10 \\
\hline
\end{tabular}

\begin{tabular}{|c|c|c|c|c|c|c|c|c|}
\hline TRN & $\begin{array}{l}\text { NOISE } \\
{[\mathrm{m}] 1-\sigma}\end{array}$ & $\begin{array}{l}\text { BIAS } \\
{[\mathrm{m}] 1-\sigma}\end{array}$ & $\begin{array}{l}\text { LATITUDE } \\
\text { MAP BIAS } \\
\text { [deg] 1- } \sigma\end{array}$ & 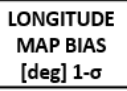 & $\begin{array}{l}\text { FIELD OF VIEW } \\
\text { [deg] }\end{array}$ & $\begin{array}{c}\text { SAMPLE } \\
\text { RATE } \\
\text { [sec] }\end{array}$ & $\begin{array}{c}\text { MIN } \\
\text { ALTITUDE } \\
{[\mathrm{m}]}\end{array}$ & $\begin{array}{c}\text { MAX } \\
\text { ALTITUDE } \\
\text { [m] }\end{array}$ \\
\hline GENERIC & {$\left[\begin{array}{llll}60 & 30 & 30\end{array}\right]$} & 5 & $1 e-4$ & $1 \mathrm{e}-4$ & 60 & 10 & 100 & $10 \mathrm{e} 3$ \\
\hline
\end{tabular}

\begin{tabular}{|c|c|c|c|c|c|c|c|c|c|c|}
\hline $\begin{array}{c}\text { RADAR } \\
\text { ALTIMETER }\end{array}$ & $\begin{array}{l}\text { TOPOGRAPHIC } \\
\text { MARKOV } \\
\text { [m] 1- } \sigma\end{array}$ & $\begin{array}{c}\text { SCALE FACTOR } \\
1-\sigma\end{array}$ & $\begin{array}{c}\text { MISALIGNMENT } \\
\text { BIAS } \\
\text { [deg] 1-б }\end{array}$ & $\begin{array}{c}\text { TOPOGRAPHIC } \\
\text { MARKOV TAU } \\
\text { [sec] }\end{array}$ & $\begin{array}{c}\text { TOPOGRAPHIC } \\
\text { MARKOV Q } \\
1-\sigma\end{array}$ & $\begin{array}{l}\text { MEASUREMENT } \\
{[\mathrm{m}] 1-\sigma}\end{array}$ & $\begin{array}{l}\text { FIELD OF } \\
\text { VIEW } \\
\text { [deg] }\end{array}$ & $\begin{array}{l}\text { SAMPLE } \\
\text { RATE } \\
\text { [sec] }\end{array}$ & $\begin{array}{c}\text { MIN } \\
\text { ALTITUDE } \\
{[\mathrm{m}]}\end{array}$ & $\begin{array}{c}\text { MAX } \\
\text { ALTITUDE } \\
\text { [m] }\end{array}$ \\
\hline HG8500 & 1 & 5.00E-06 & $1.00 \mathrm{E}-04$ & 60 & 0.01 & 1 & 35 & 10 & 20 & $2.44 \mathrm{e} 3$ \\
\hline
\end{tabular}

LN200S IMU sensor specs (blue shaded cells) are extracted from Northrop Grumman LN200s spec sheet Sodern Star Tracker sensor specs (blue shaded cells) are based on Sodern spec sheet HG8500 Radar Altimeter sensor specs (blue shaded cells) are extracted from Honeywell hg8500 spec sheet

Table 1. Example of Sensor Specification for the Conceptual Lander 


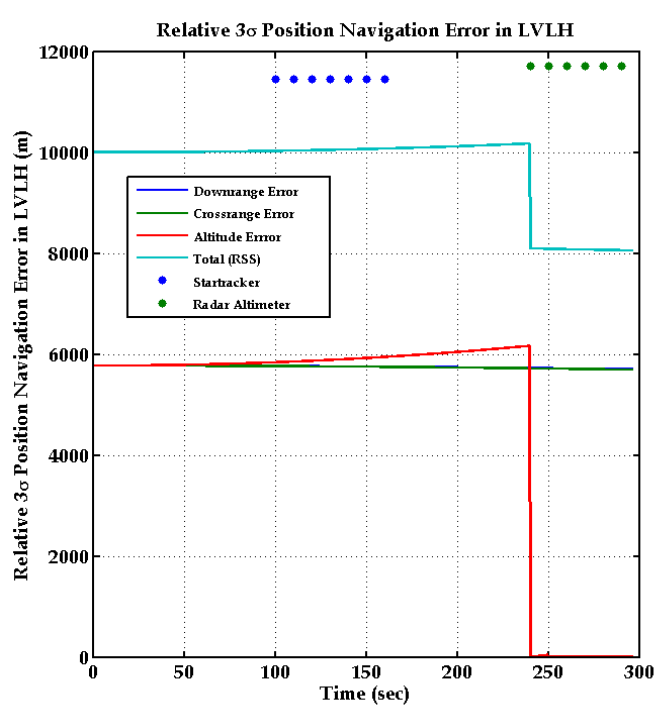

(a)

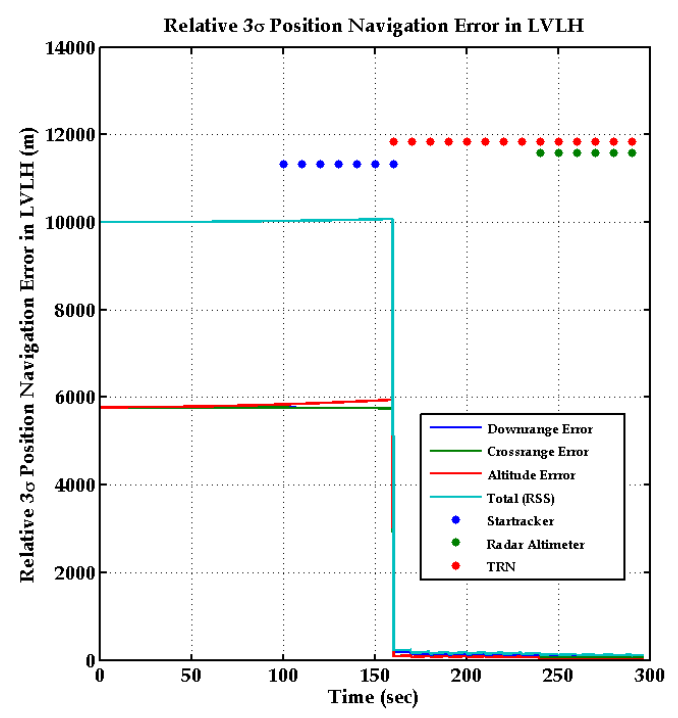

(b)

Figure 1. IMU, Star Tracker, Radar Altimeter, and TRN Sensors for a Conceptual Lander

\section{Linear Covariance Analysis Overview}

In general, to perform the necessary analysis and evaluation of a GNC system, there are several key variables of interest, including environment dispersions, navigation dispersions, actual navigation error, and onboard navigation error ${ }^{14}$. These variables characterize the performance of the system and are used to develop and validate mission objectives and requirements. Extensive effort and resources are often allocated for the very purpose of producing and analyzing the quantities of these system parameters. For this paper, they are instrumental in characterizing the overall system and navigation performance for descending to the surface of the moon. This section is dedicated to formally defining these metrics and summarizing the analysis techniques.

\section{Performance Metrics}

The environment dispersions $\delta \mathbf{x}$ are defined as the difference between the environment state $\mathbf{x}$ and the nominal state $\overline{\mathbf{x}}$. The environment state is an $(n \times 1)$ vector that represents the true or actual state. The nominal state is also an $(n \times 1)$ vector that represents the desired or reference state. The covariance of the environment dispersions, $\mathbf{D}$, indicates how precisely the system can follow a desired trajectory, as shown in Eq. (7)

$$
\delta \mathbf{x} \equiv \mathbf{x}-\overline{\mathbf{x}}, \quad \mathbf{D}=E\left[\delta \mathbf{x} \delta \mathbf{x}^{T}\right]
$$

The environment (or true) dispersions are often referred to as simply dispersions (e.g., trajectory dispersions, position dispersions, relative dispersions).

The navigation dispersions are defined as the difference between the navigation state $\hat{\mathbf{x}}$ and the nominal state, as shown in Eq. (8). The navigation state is an $(\hat{n} \times 1)$ vector that represents the estimated state.

$$
\delta \hat{\mathbf{x}} \equiv \hat{\mathbf{x}}-\mathbf{N} \overline{\mathbf{x}}, \quad \hat{\mathbf{D}}=E\left[\delta \hat{\mathbf{x}} \delta \tilde{\mathbf{x}}^{T}\right]
$$

The matrix $\mathbf{N}$ is an $(\hat{n} \times n)$ mapping matrix that defines the estimated state in terms of the true and nominal state. It typically cancels the attitude rate state when gyro measurements are incorporated in lieu of an angular rate estimate. An inverse mapping takes the estimated state to the true state, represented with an $(n \times \hat{n})$ matrix $\mathbf{N}^{T}$. The 
covariance of the navigation dispersions, $\hat{\mathbf{D}}$, reflects how precisely the onboard system thinks it can follow a prescribed reference trajectory.

The true navigation error is the difference between the environment and navigation states. It is also the difference between the environment and the navigation dispersions. The covariance of the true navigation error, $\mathbf{P}$, shown in in Eq. (9), characterizes how precisely the onboard navigation system can determine the actual state.

$$
\delta \mathbf{e} \equiv \mathbf{N} \mathbf{x}-\hat{\mathbf{x}}=\mathbf{N} \delta \mathbf{x}-\delta \hat{\mathbf{x}}, \quad \mathbf{P}=E\left[\delta \mathbf{e} \delta \mathbf{e}^{T}\right]
$$

The onboard navigation error is never computed but is used to develop the onboard navigation filter equations. It is defined as the difference between the design state, $\mathbf{x}$, and the navigation state, as shown in Eq. (10)

$$
\delta \hat{\mathbf{e}} \equiv \mathbf{x}-\hat{\mathbf{x}}, \quad \hat{\mathbf{P}}=E\left[\delta \hat{\mathbf{e}} \delta \hat{\mathbf{e}}^{T}\right]
$$

The covariance of the onboard navigation error, $\hat{\mathbf{P}}$, shows how precisely the onboard navigation system thinks it can determine the actual state. The performance of the onboard navigation system is determined by comparing $\hat{\mathbf{P}}$ to the actual navigation performance $\mathbf{P}$. It is the covariance of the true dispersions, navigation dispersions, true navigation error, and the onboard navigation error that are ultimately used to analyze and assess the performance of a proposed GNC system.

\section{Obtaining the Performance Metrics}

A common approach to obtain these performance metrics is to use a Monte Carlo simulation as shown in Figure 2, where the sample statistics of hundreds or thousands of runs are used to numerically compute the desired covariance matrices, as shown in Eq. (11)

$$
\begin{array}{ll}
\mathbf{D}=\frac{1}{N-1} \sum \delta \mathbf{x} \delta \mathbf{x}^{T}, & \hat{\mathbf{D}}=\frac{1}{N-1} \sum \delta \hat{\mathbf{x}} \delta \tilde{\mathbf{x}}^{T} \\
\mathbf{P}=\frac{1}{N-1} \sum \delta \mathbf{e} \delta \mathbf{e}^{T}, & \hat{\mathbf{P}}=\hat{\mathbf{P}}
\end{array}
$$

This consistent statistical information can be obtained using linear covariance analysis techniques ${ }^{15,16,17,18}$ by directly propagating, updating, and correcting an augmented state covariance matrix $\mathbf{C}$,

$$
\mathbf{C}=E\left[\delta \mathbf{X} \delta \mathbf{X}^{T}\right]
$$

where the augmented state $\delta \mathbf{X}^{T}=\left[\begin{array}{ll}\delta \mathbf{x}^{T} & \delta \tilde{\mathbf{x}}^{T}\end{array}\right]$ consists of the true dispersions and the navigation dispersions. Notice that by simply pre- and post-multiplying the augmented state covariance matrix by the following matrices, the covariance matrices for the trajectory dispersions, navigation dispersions, and the navigation error can be obtained, as shown in Eq. (13)

$$
\begin{array}{ll}
\mathbf{D}=\left[\mathbf{I}_{n x x}, \mathbf{0}_{\mathrm{nxn}} \mathbf{N}^{T}\right] \mathbf{C}\left[\mathbf{I}_{n x n}, \mathbf{0}_{\mathrm{nxx}} \mathbf{N}^{T}\right]^{\mathrm{T}}, & \hat{\mathbf{D}}=\left[\mathbf{N} \mathbf{0}_{\mathrm{n} \times \mathrm{n}}, \mathbf{I}_{\hat{n} \times \hat{n}}\right] \mathbf{C}\left[\mathbf{N} \mathbf{0}_{\mathrm{nxn}}, \mathbf{I}_{\hat{n} x \hat{n}}\right]^{\mathrm{T}} \\
\mathbf{P}=\left[\mathbf{N I}_{n x n},-\mathbf{I}_{\hat{n} x \hat{n}}\right] \mathbf{C}\left[\mathbf{N} \mathbf{I}_{\mathrm{n} \times \mathrm{n}},-\mathbf{I}_{\hat{n} x \hat{n}}\right]^{\mathrm{T}}, & \hat{\mathbf{P}}=\hat{\mathbf{P}}
\end{array}
$$

Consequently, Monte Carlo and linear covariance analysis techniques provide a complimentary analysis package since they each generate the consistent statistical information using different approaches. The strengths and weaknesses of one technique often offset the other. Such is the case when developing and analyzing the preliminary trajectory design and concept of operations for close-proximity asteroid operations. 


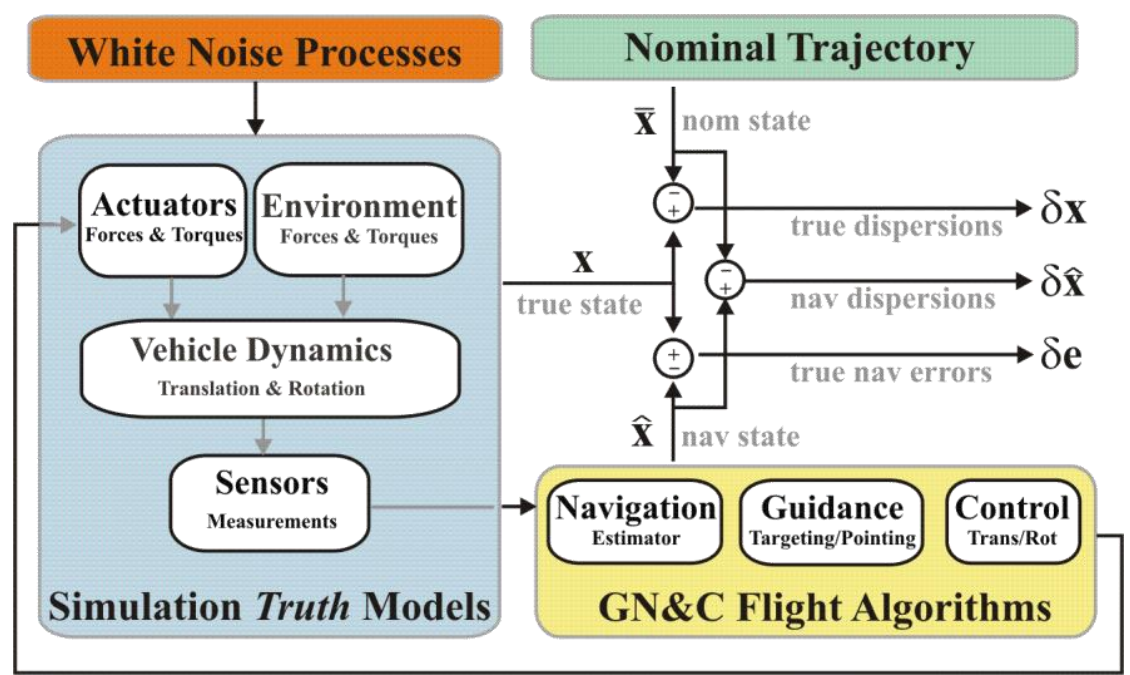

Figure 2. GN\&C Performance Metrics in a General Monte Carlo Simulation

\section{Linear Covariance Analysis for Lunar Lander}

LinCov is designed to estimate navigation errors and trajectory dispersions of a vehicle GNC system for space missions. Based on linear covariance theory, a single LinCov run can give a similar estimation of trajectory and navigation performance as that of thousands of Monte Carlo simulations. In this paper, a LinCov analysis will be used to identify candidate sensor suites as well as initial relative state dispersion requirements necessary for satisfying precision landing requirements. Typical precision landing requirements consist a landing site footprint, vertical and horizontal landing velocities, as well as the attitude and rate.

The Draper LinCov tool provides the following analysis capabilities: (1) Nominal analysis, which predicts the nominal trajectory performance, (2) Dispersion analysis, which predicts the Trajectory and Navigation dispersed performance for the pre-selected sensor suites on the lunar lander, (3) Sensitivity analysis, which estimates the sensitivity of the overall Trajectory and Navigation dispersion performance to different components (sensors, environments, and initial conditions) and their corresponding parameters, and (4) Requirement analysis, which will help GNC and systems engineers to finalize the sensor selection and determine the corresponding sensor specifications to meet the landing and budget requirements. In this section, Nominal, Dispersion, and Sensitivity analysis will be performed for the conceptual lunar lander GNC design.

In the nominal analysis, a nominal trajectory and GNC are installed in the LinCov tool. The implementation is verified by comparing the LinCov nominal response to a $6 \mathrm{DOF}$ nonlinear simulation. Both translational and rotational states will be verified. Figure 3 shows the time history data of an example lunar mission which consists of three flight phases: (1) brake burn phase from $0 \mathrm{sec}$ to $\sim 81 \mathrm{sec}$, (2) coast phase from $\sim 81 \mathrm{sec}$ to $\sim 160 \mathrm{sec}$ and (3) approach and landing phase from $\sim 160 \mathrm{sec}$ to $\sim 296 \mathrm{sec}$. In this example, the IMU is active during all three flight phases. Figure 4 demonstrates the sensor activation timeline. In this example, star tracker is used only during coast phase. The TRN sensor is activated when altitude is below $10 \mathrm{~km}$ and altimeter is turned on when altitude is less than $2.4 \mathrm{~km}$. 

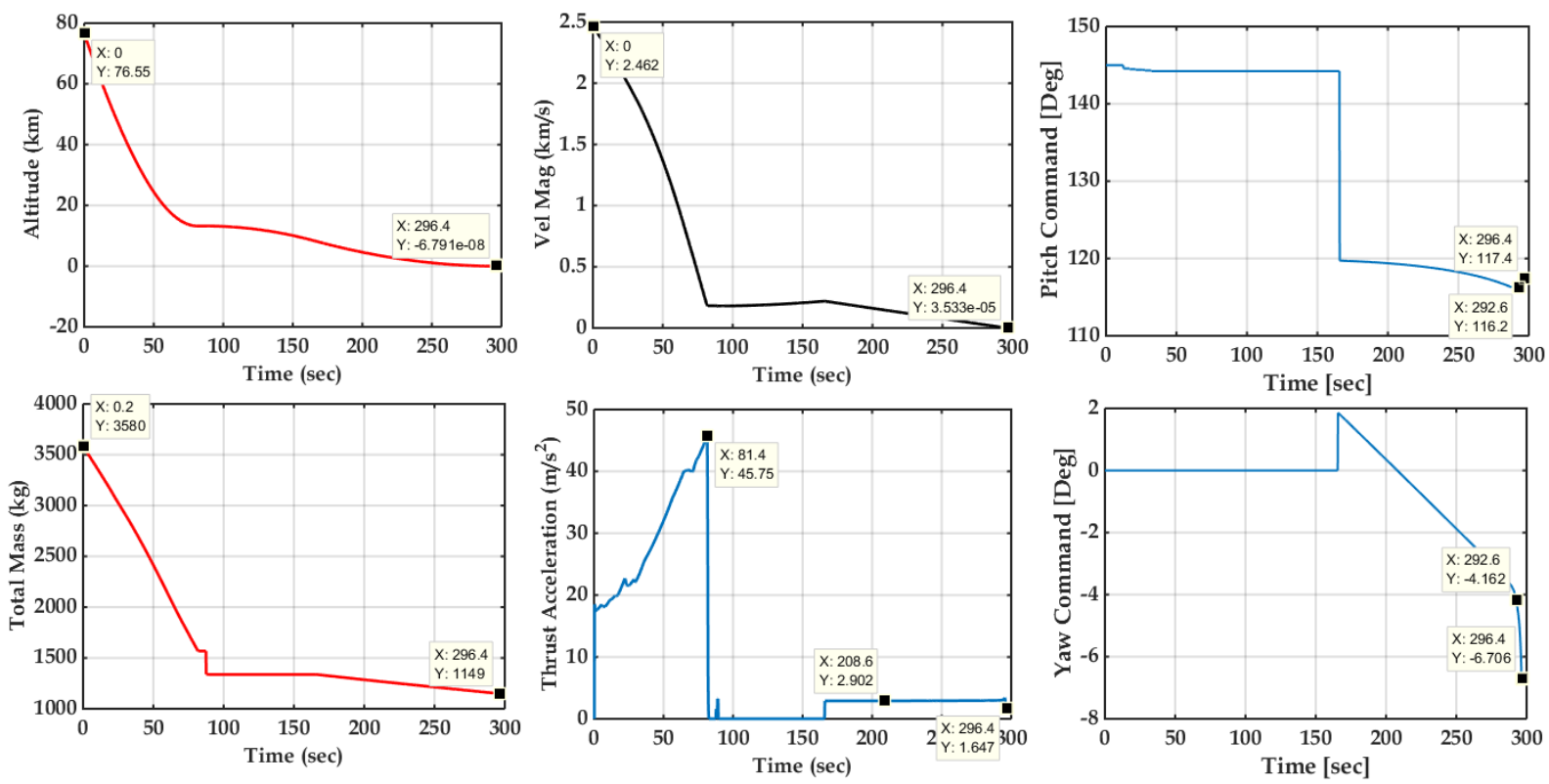

Figure 3. An Example 6-DOF Nominal Response

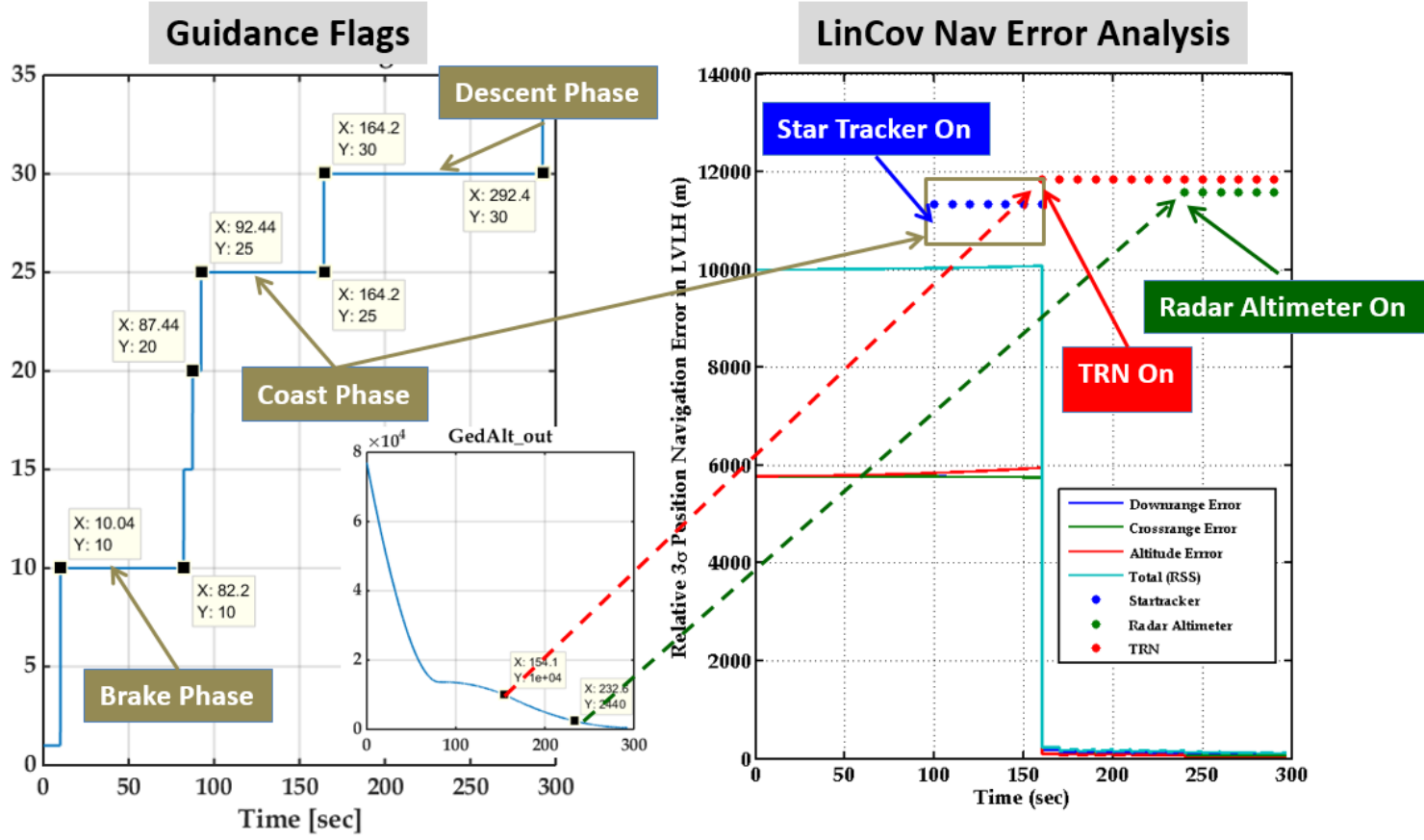

Figure 4. An Example of Sensor Activation Time Line

Based on the linear covariance theory introduced in the previous section, sensor error specs, and initial navigation errors and trajectory dispersions for this lunar mission example, the navigation error and trajectory dispersions can be estimated using LinCov. The translational state dispersion analysis for this example are demonstrated in Figure 5. The green line in Figure 5 is the prediction of the time history of the onboard navigation translational state error. In this example, the position navigation error is dramatically reduced with the first TRN 
estimate and altitude navigation estimation is improved when the altimeter sensor turns on. The position and velocity error dispersion results shown in Figure 5 indicate that the translational states are controlled via an openloop guidance during the braking phase. The corresponding state errors are corrected via a closed-loop guidance during the Approach and Landing phase. The rotational states dispersion analysis for this example are demonstrated in Figure 6. The rate error is always zero since the model replacement method is assumed. The attitude error is significantly reduced when the star tracker sensor is activated.
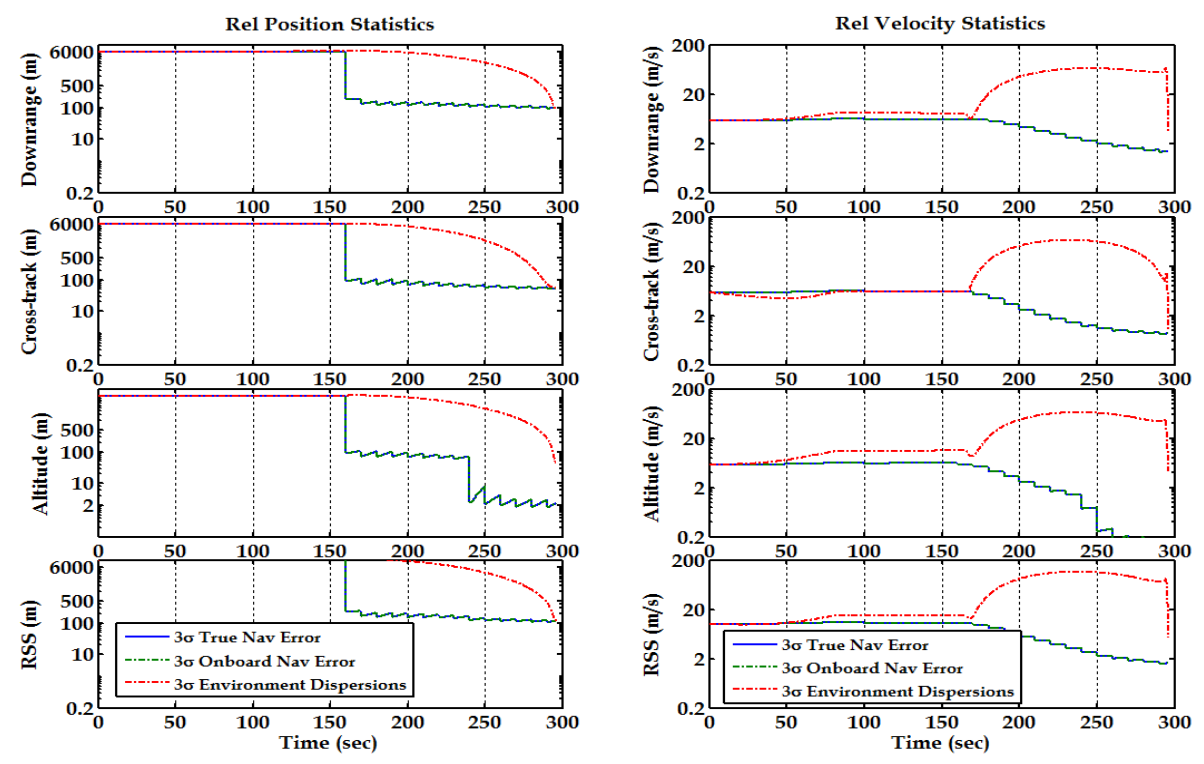

Figure 5. An Example of Navigation Error and Trajectory Dispersion Estimation for Translational States
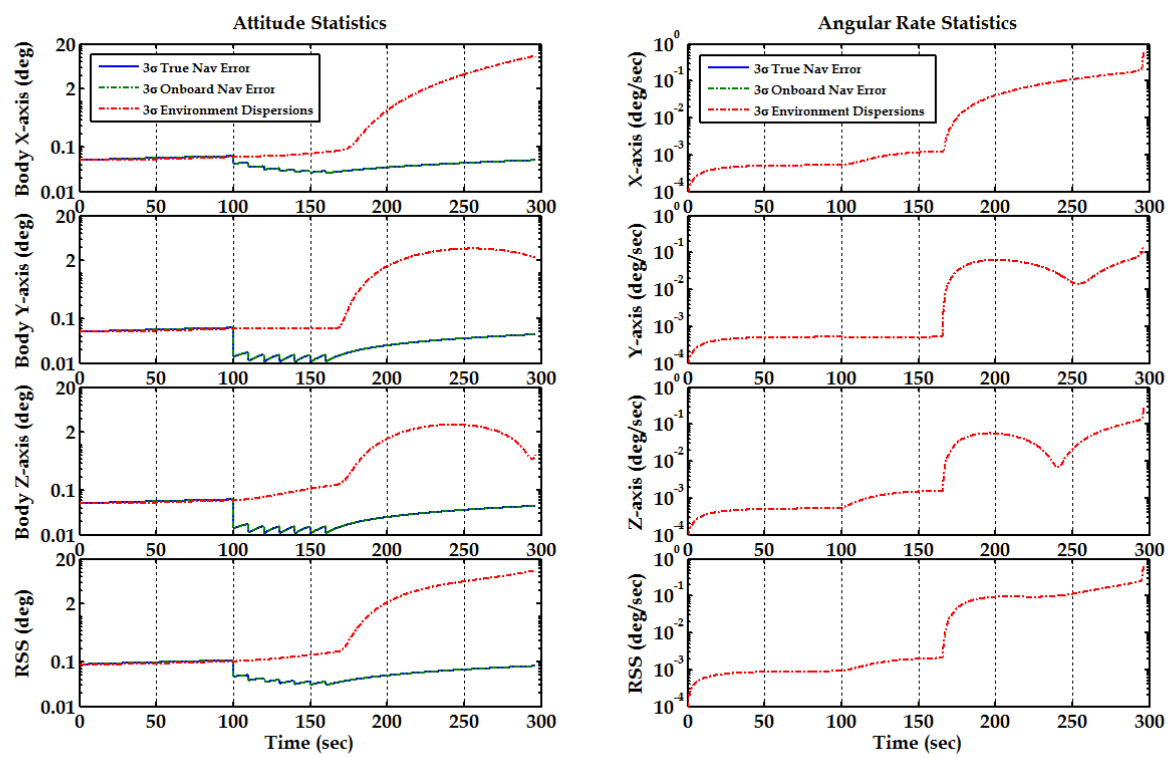

Figure 6. An Example of Navigation Error and Trajectory Dispersion Estimation for Rotational States 
The dispersed propellant and Delta-V usage are obtained as follows. The expelled propellant mass, $m_{\text {expelled }}$, is included as a state using the equation of motion shown in Eq. (14)

$$
\dot{m}_{\text {expelled }}=\frac{m_{\text {init }}-m_{\text {expelled }}}{I_{s p} g_{0}}\|\mathbf{a}\|
$$

where $m_{\text {expelled }}$ is zero at time zero, $m_{\text {init }}$ is the total initial vehicle mass (including propellant), $I_{s p}$ is the thruster specific impulse, $g_{0}$ is the Earth gravitational constant, and a is the control acceleration. Therefore, the Delta-V usage can be calculated by Eq. (15)

$$
\Delta v \approx \Delta m_{\text {expelled }} \frac{I_{s p} g_{0}}{m_{\text {init }}-m_{\text {expelled }}} \equiv \Delta m_{\text {expelled }} \mathrm{H}
$$

Recalling Eq. (7), let the expelled mass environment dispersion element of the covariance be given by Eq. (16)

$$
\mathrm{D}_{m}=E\left[\delta m_{\text {expelled }} \delta m_{\text {expelled }}^{T}\right]
$$

Then the Delta-V dispersion covariance is given by Eq. (17)

$$
\mathrm{D}_{\Delta v}=E\left[\delta \Delta v \delta \Delta v^{T}\right]=\mathrm{HD}_{m} \mathrm{H}^{T}
$$

The consumed propellant and Delta-V are shown in Figure 7. The right-hand side plots in Figure 7 illustrate the nominal propellant consumption and Delta- $\mathrm{V}$ costs. The error budgets as a function of time for both propellant and Delta-V are shown on the left-hand side of Figure 7.
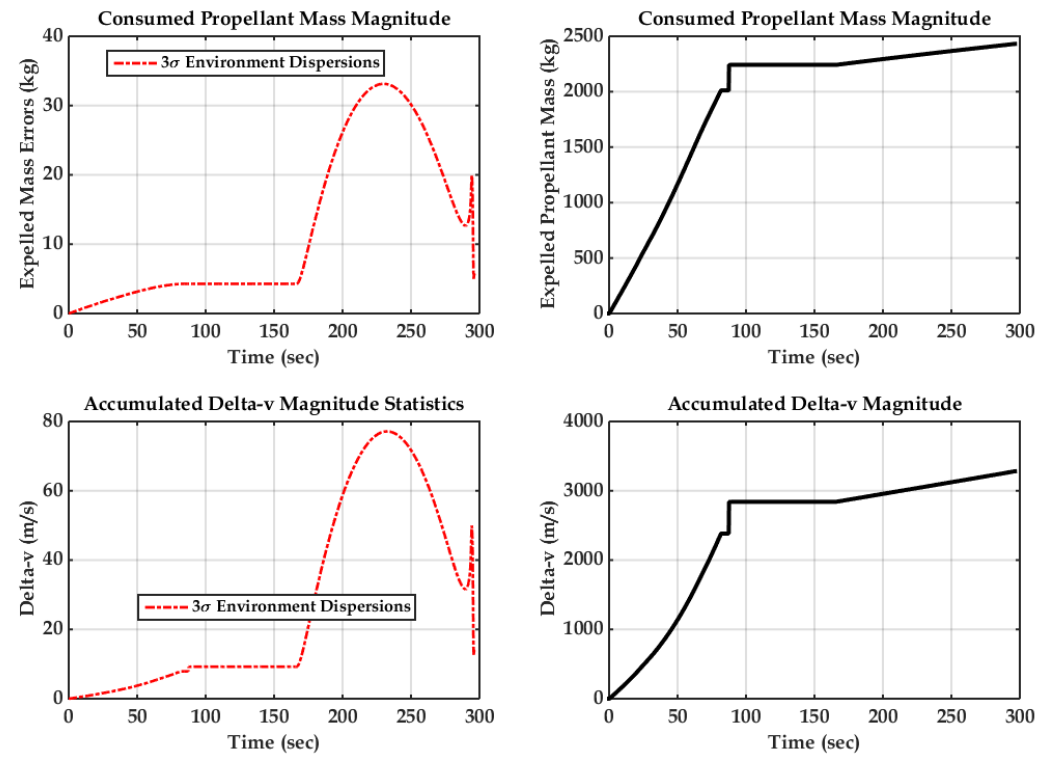

Figure 7. An Example of Expelled Mass Errors and Delta-V Estimation

The dispersion analysis shown in Figure 5 to Figure 7 estimates the navigation error and trajectory dispersion for the entire mission. From these plots, users can predict worst-case navigation performance and trajectory deviation at any flight time. GNC engineers can use this data to predict the flight performance against requirements. In this 
lander design example, the conceptual mission requires that GNC shall place the lander within 100m circular landing foot print of the prelaunch selected landing site, maintain a horizontal and horizontal velocity of less than 2 $\mathrm{m} / \mathrm{s}$ at touchdown, and control the spacecraft angle rate to less than $2 \mathrm{deg} / \mathrm{sec}$ in all three axes at touchdown. If the dispersion analysis indicates that any mission landing site requirement is not satisfied, further analysis can be performed with LinCov to obtain more information. In this example, the baseline sensors and initial dispersion configuration violates the landing velocity requirement.

Sensitivity analysis can be used to identify the dominant sensor parameters and/or initial states that are the key factors which result in landing site performance violations by performing dispersion analysis with only one error source active at a time. Figure 8 to Figure 11 demonstrate the sensitivity analysis for position navigation error, velocity navigation error, position trajectory dispersion and velocity trajectory dispersion, respectively. The case with all errors turned on ("All") and all turned off ("Zero") are shown for reference, along with cases for initial condition errors and each sensor error, and "Others" which includes environmental process noise to account for unmodeled disturbances. Because the error sources are assumed to be independent, the root-sum-square ("RSS") of all the cases together matches the "All" case. In these example analyses, the TRN sensor and initial conditions lead to the large landing site errors. A requirement analysis will be performed next to search for candidate TRN sensor specs along with initial relative states dispersion range for Lander GNC to meet landing site requirements. The detailed requirement results are provided in the following section.
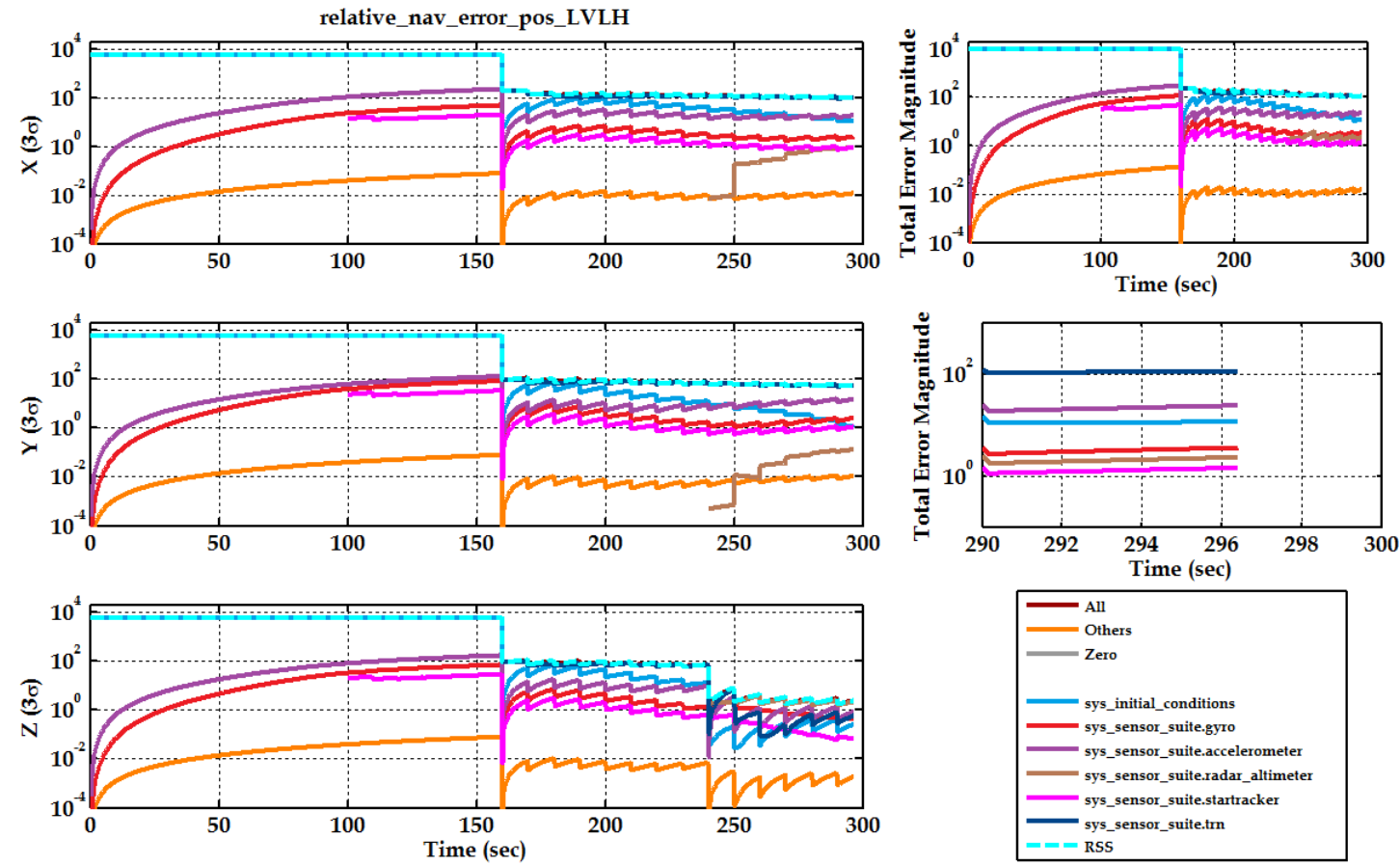

Figure 8. An Example of Navigation Error Sensitivity Analysis for Position States 

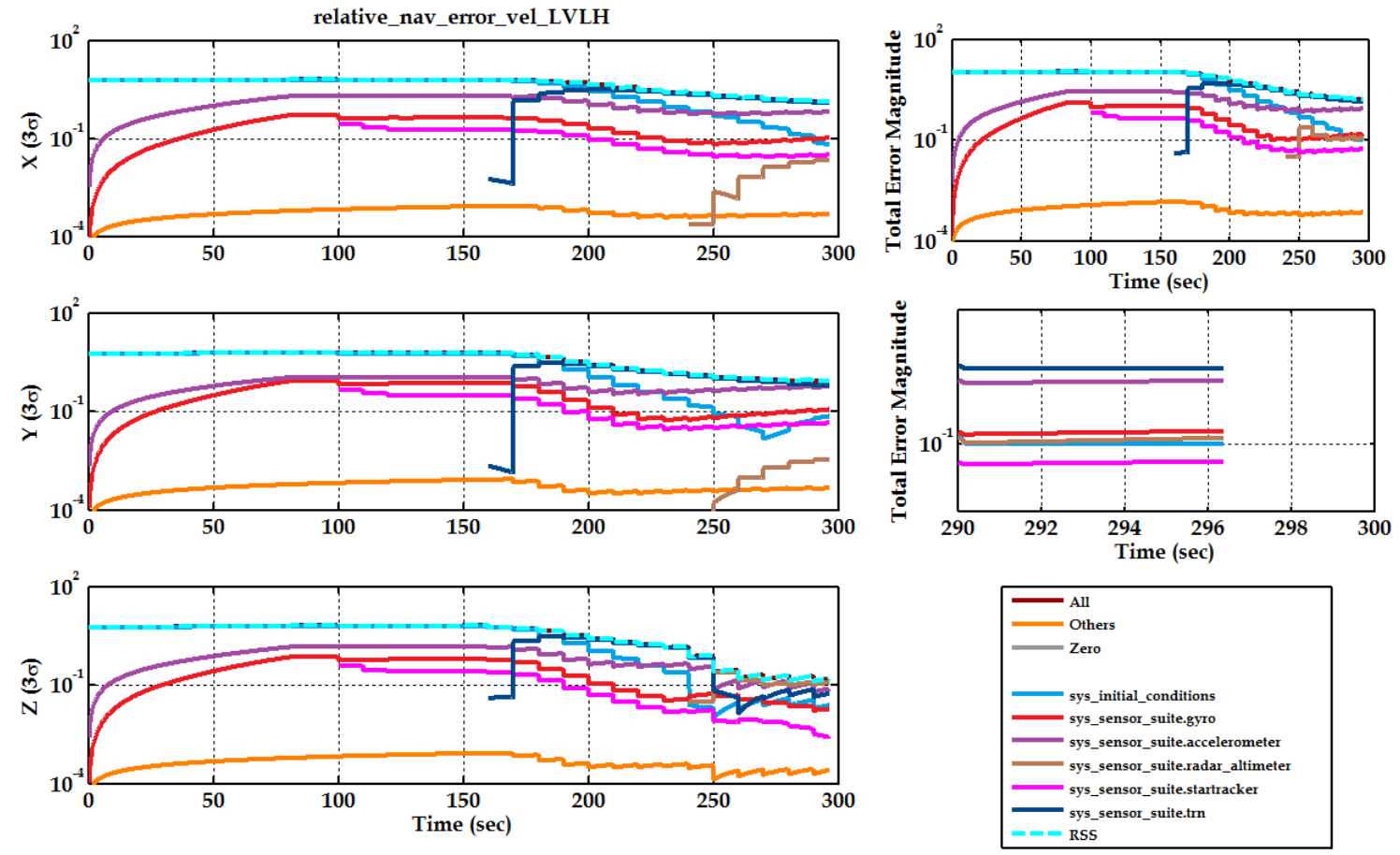

Figure 9. An Example of Navigation Error Sensitivity Analysis for Velocity States
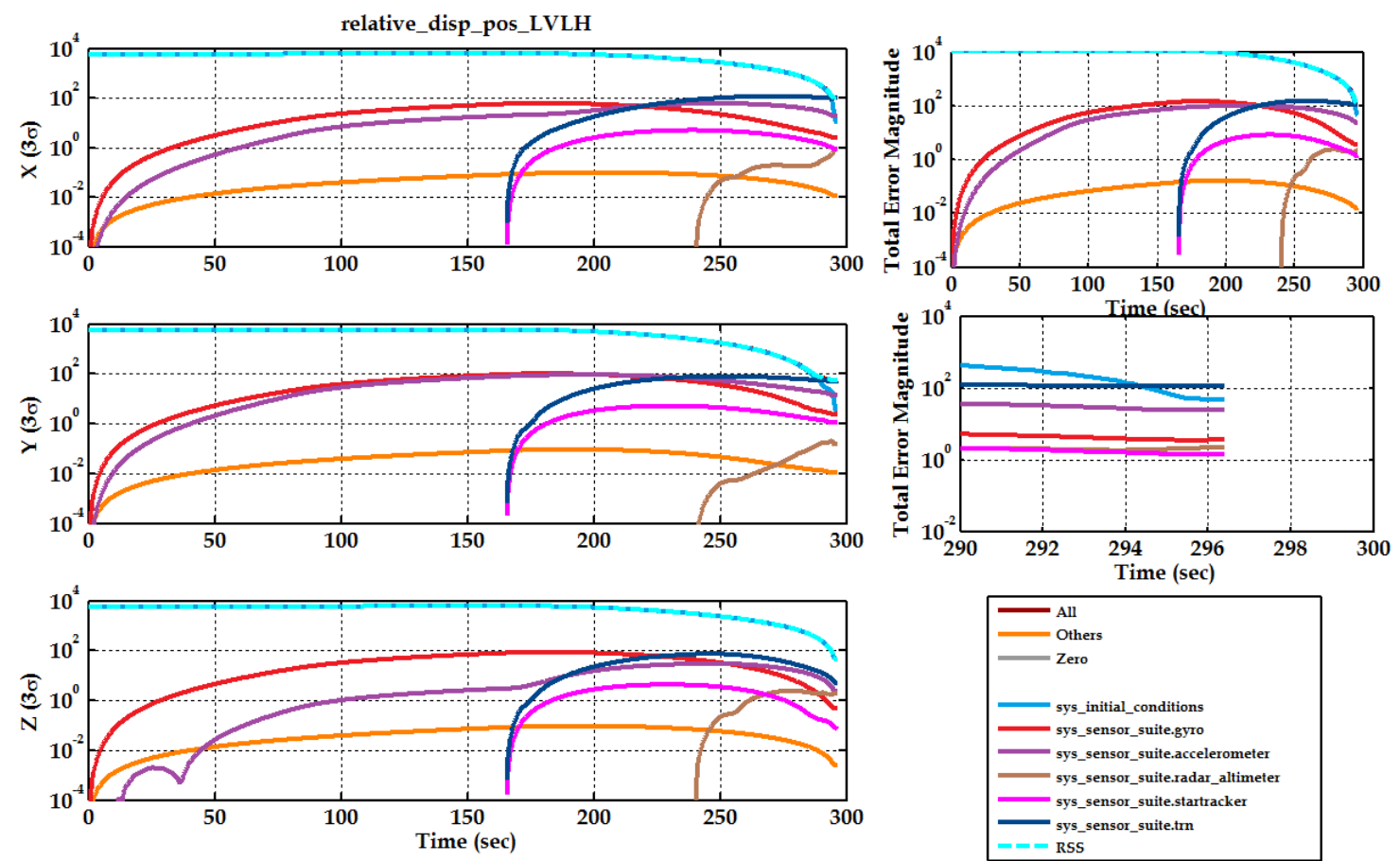

Figure 10. An Example of Trajectory Dispersion Sensitivity Analysis for Position States 

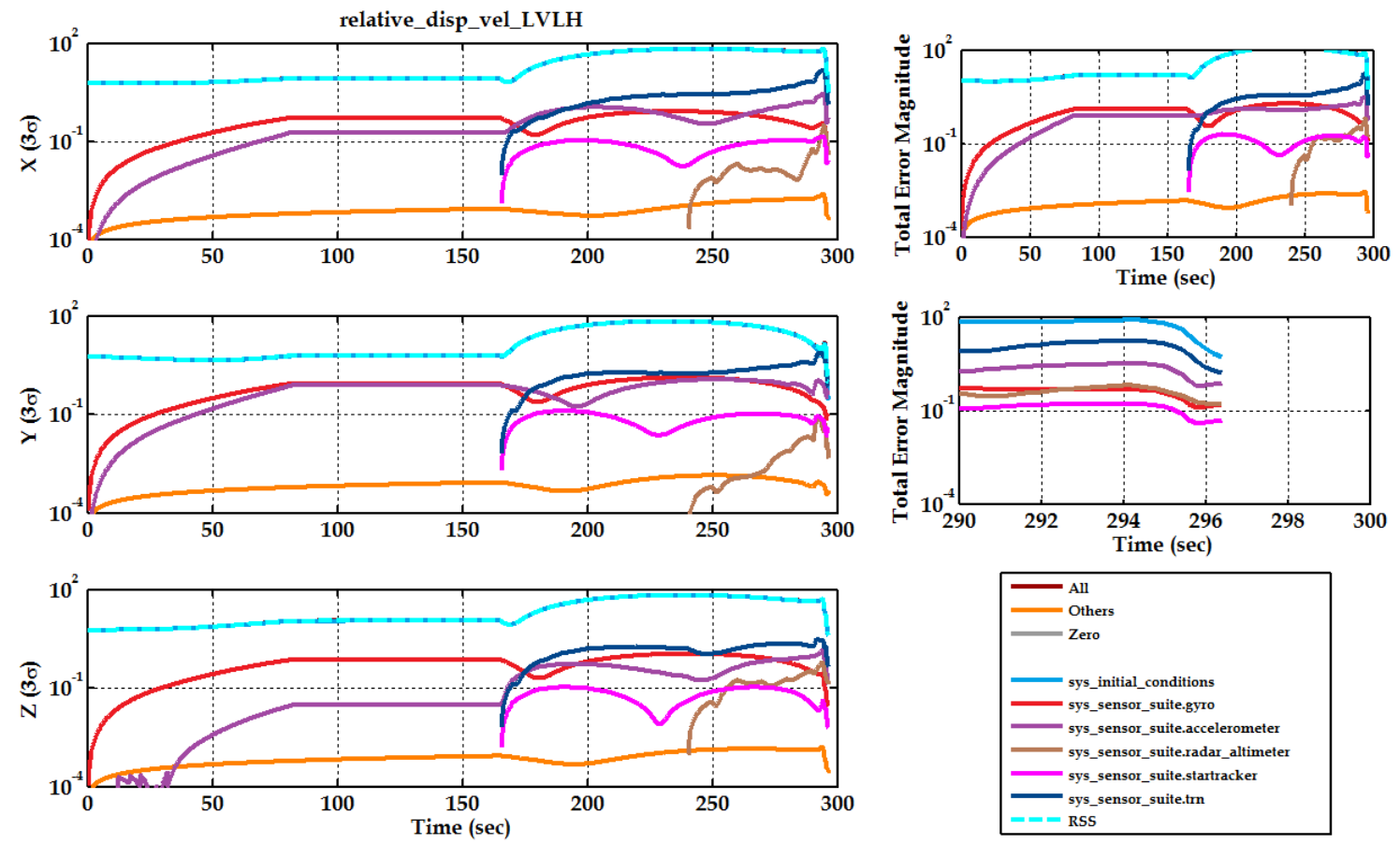

Figure 11. An Example of Trajectory Dispersion Sensitivity Analysis for Velocity States

VI. Final Sensor Selection for Lunar Lander

As demonstrated in the previous section, the baseline sensors and initial dispersion configuration defined in Section III violates the landing velocity accuracy requirement. The position requirement is marginally met using perfect actuators. Landing site velocity dispersions are dominated by initial conditions followed by TRN measurement accuracy. In this section, a LinCov Requirement Analysis is performed to redesign initial dispersion and TRN noise/error characteristics such that the landing site requirement will be met.

An example performance analysis is summarized in Table 2. The results suggest that (1) single beam altimeter cannot be used to improve downrange and cross track landing site accuracy, (2) reduced TRN noise can cut down landing site position drift but not velocity drift, (3) baseline TRN bias and map bias has little impact on the landing accuracy, and (4) reducing initial position dispersion will improve the landing site velocity performance but not vice versa.

\begin{tabular}{|c|c|c|c|c|c|c|c|}
\hline \multirow[b]{2}{*}{ Parameter } & \multirow[b]{2}{*}{ Variation From Baseline } & \multicolumn{3}{|c|}{ Position (meter) } & \multicolumn{3}{|c|}{ Velocity $(\mathrm{m} / \mathrm{s})$} \\
\hline & & $\begin{array}{l}\text { Downrange } \\
(100 \mathrm{~m} 3 \sigma)\end{array}$ & $\begin{array}{l}\text { Cross Track } \\
(100 \mathrm{~m}, 3 \sigma)\end{array}$ & Altitude & $\begin{array}{l}\text { Downrange } \\
(2 \mathrm{~m} / \mathrm{s} 3 \sigma)\end{array}$ & $\begin{array}{l}\text { Cross Track } \\
(2 \mathrm{~m} / \mathrm{s}, 3 \sigma)\end{array}$ & $\begin{array}{c}\text { Altitude } \\
(2 \mathrm{~m} / \mathrm{s}, 3 \sigma)\end{array}$ \\
\hline Baseline & & 98.73 & 54.05 & 44.71 & 3.25 & 0.96 & 4.20 \\
\hline \multirow{5}{*}{ TRN } & Turn off TRN & 5700.00 & 5693.00 & 76.57 & 6.98 & 4.86 & 7.29 \\
\hline & Reduced Baseline Bias by a factor of 5 & 97.64 & 52.02 & 44.11 & 3.25 & 0.96 & 4.19 \\
\hline & Reduce Baseline Map Bias by a factor of 10 & 98.73 & 53.29 & 44.11 & 3.25 & 0.96 & 4.19 \\
\hline & Reduced Baseline Noise by $25 \%$ & 75.99 & 43.24 & 43.99 & 3.13 & 0.85 & 4.18 \\
\hline & Reduced Baseline Noise by a factor of 4 & 31.66 & 23.99 & 43.81 & 2.96 & 0.59 & 4.16 \\
\hline \multirow{4}{*}{$\begin{array}{c}\text { Initial States } \\
\text { Dispersion }\end{array}$} & Reduce Baseline Init. Pos by a factor of 10 & 98.40 & 53.95 & 16.14 & 1.80 & 0.90 & 1.54 \\
\hline & Reduce Baseline Init. Vel by a factor of $\mathbf{1 0}$ & 96.39 & 54.00 & 43.54 & 3.21 & 0.94 & 4.14 \\
\hline & Reduce Baseline Init. Pos/Vel by a factor of 10 & 96.19 & 53.90 & 14.53 & 1.73 & 0.88 & 1.37 \\
\hline & Reduce Baseline Init. Pos/Vel by a factor of 100 & 93.86 & 53.72 & 6.04 & 1.45 & 0.86 & 0.54 \\
\hline
\end{tabular}

Table 2. An Example Requirement Analysis 
Converting top level system requirements into low level navigation requirements has traditionally been an adhoc art relying on engineering judgment, experience, and rough calculations. Table 2 is an example of how LinCov analysis brings rigor to this effort. In this approach, each identified key parameter is manually adjusted until all navigation requirements are satisfied.

Deriving and validating navigation requirements for a specific application can, however, become an iterative and laborious task with only subjective results. By combining and applying fundamental principles commonly accepted with Monte Carlo analysis, linear covariance analysis, and sensitivity analysis; a fast, practical, and reliable methodology for deriving and validating navigation requirements emerges that also identifies the optimal sensor suite to fulfill those requirements. Instead of fully incorporating sensor models and filter algorithms to capture the performance of the navigation system, the navigation performance can be replicated using stochastic navigation. Sensitivity analysis can then provide a quick and automated approach for determining the navigation requirements. Once the sensitivity data is generated, the total uncertainty given different scaled values of the original error sources can be quickly obtained without re-running the simulation ${ }^{19}$. The requirement generation capability introduced in reference 19 is more efficient than Table 2 approach.

In the final requirement analysis, $3 \mathrm{~dB}$ margins are reserved for thruster mounting errors and firing delays. This is equivalent to a reduced landing site footprint and touch down velocity requirement by $30 \%$. Figure 12 illustrates the touchdown performance with the improved TRN sensor noise error spec and tighter initial position dispersion. The blue curve in the crossrange vs. downrange plot represents the nominal trajectory of the lander while the red ellipse describes the 3- $\sigma$ dispersion of the footprint. With the improved TRN sensor noise error spec and tighter initial position dispersion, both position and velocity landing site requirements are met.
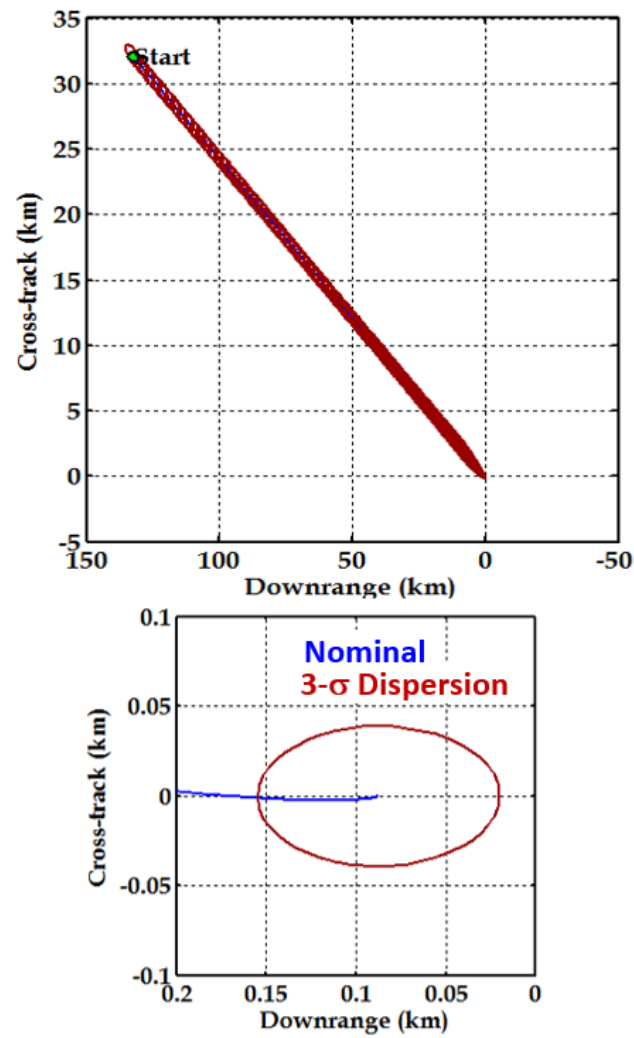
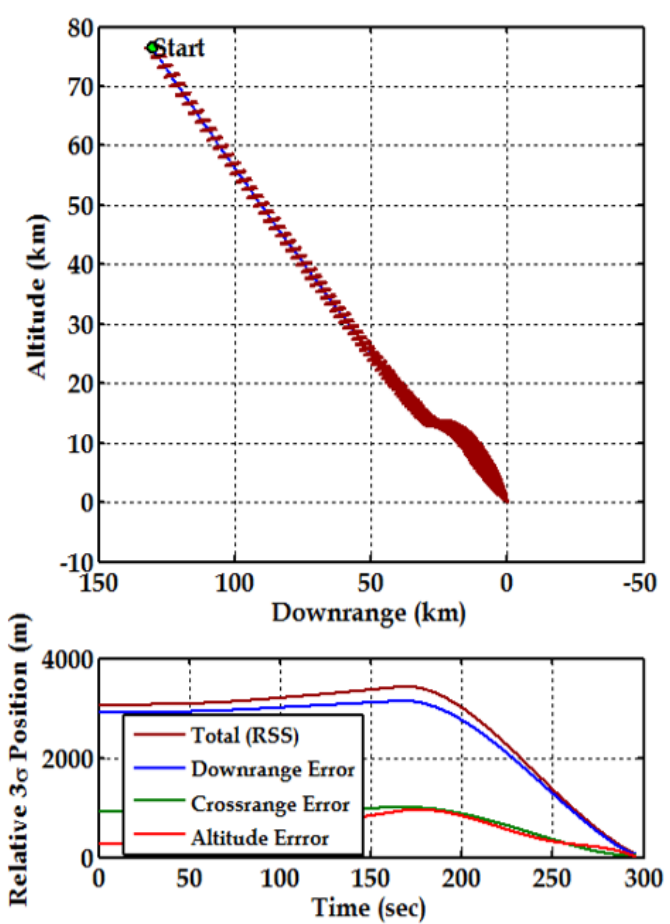

Figure 12. An Example of Landing Site Footprint 


\section{Summary}

In this paper, a conceptual lunar lander and a corresponding GNC architecture and flight control algorithms are introduced. The theory and application of LinCov are reviewed. A LinCov analysis for the conceptual lunar lander GNC is performed for sensor selection. The sensitivity analysis is performed to identify which sensor specifications drive the landing site requirement violation. The LinCov requirement analysis is then performed to determine the corresponding sensor specifications to meet the landing performance and budget requirements.

\section{References}

1 Andrews, D., Colaprete, A., Quinn, J., Chavers, D., and Picard, M., "Introducing the Resource Prospector (RP)," AIAA Space Conferences, San Diego, CA, 2014, AIAA-2014-4378.

2 Geller, D. K., "Linear Covariance Techniques for Orbital Rendezvous Analysis and Autonomous Onboard Mission Planning," Journal of Guidance, Control, and Dynamics, Vol. 29, No. 6, 2006, pp. 1404-1414.

3 Geller, D. K., and Christensen, D., "Linear Covariance Analysis for Powered Lunar Descent and Landing," Journal of Spacecraft and Rockets, Vol. 46, No. 6, Nov-Dec 2019.

4 Bennett, F. V.,"Apollo Experience Report - Misssion Planning for Lunar Module Descent and Ascent," NASA Report TN D6846, 1972

5 Jang, J., Alaniz, A., Hall, R., Bedrossian, N., Hall, C., Ryan, S., Jackson, M., “Ares I Flight Control System,” AIAA GN\&C Conference, Toronto, Canada, 2010, AIAA-2010-8442.

6 Penchuk, A., Hattis, P., and Kubiak, E., “A Frequency Domain Stability Analysis of a Phase Plane Control System,” Journal of Guidance, Vol. 8, No. 1, 1984

7 Wertz, J., Spacecraft Attitude Determination and Control, Boston, MS: D. Reidel Publishing Company, 1995.

8 Gelb, A., and Velde, W. V., Multiple-Input Describing Functions and Nonlinear System Design, New York, NY: McGrawHill Book Co., 1968.

9 Jang, J., Plummer, M., Bedrossian, N., Hall, C., Jackson, M., and Spanos, P., "Absolute Stability Analysis of a Phase Plane Controlled Spacecraft," 20th AAS/AIAA Space Flight Mechanics Meeting, AAS 10-112, San Diego, CA, February 2010.

10 Carson, J. M., Robertson, E. A., Trawny, N., and Amzajerdian, F., "Flight Testing ALHAT Precision Landing Technologies Integrated Onboard the Morpheus Rocket Vehicle," 2015 AIAA Space Conference and Exposition, AIAA 2015-4417, Pasadena, CA, August 2015.

11 Bishop, R. H., et al. "An Inertial Dual-State Estimator for Precision Planetary Landing with Hazard Detection and Avoidance,” 2016 AIAA SciTech Forum, AIAA-2016-0098, San Diego, CA, January 2016.

12 Flenniken IV, W., Wall, J., Bevly, D. "Characterization of Various IMU Error Sources and the Effect on Navigation Performance." In Proceedings of the 2005 ION GNSS, Long Beach, CA, September 13-16, 2005.

13 Osenar, M. J. "Performance of Automated Feature Tracking Cameras for Lunar Navigation." Master's thesis, Rice University, Houston, TX, April 2007.

14 Wright, C., Bhatt, S., Woffinden, D., Strube, M., D’Souza, C., "Linear Covariance Analysis for Proximity Operations around Asteroid 2008 EV5," AAS Guidance and Control Conference, Breckenridge, CO, 2015, AAS-15-094.

15 Maybeck, P. S., Stochastic Models, Estimation, and Control, Volume 1, Orlando, FL: Academic Press, Inc., 1979.

16 Gelb, A., Applied Optimal Estimation, Cambridge, MA: The M.I.T. Press, 1974.

17 Battin, R. H., An Introduction to the Mathematics and Methods of Astrodynamics, 1801 Alexander Bell Drive, Reston, VA 20191: AIAA, Inc., revised ed., 1999.

18 Geller, D. K., "Linear Covariance Techniques for Orbital Rendezvous Analysis and Autonomous Onboard Mission Planning," Journal of Guidance, Control, and Dynamics, Vol. 29, November-December 2006, pp. 1404-1414.

19 Woffinden, D., and Breger, L., "Automated Derivation and Verification of Navigation Requirements for On-orbit Rendezvous,” 2013 AIAA GNC Conference, AIAA 2013-4964, Boston, MA, August 2013. 